\title{
NONLINEAR PERIODIC PARABOLIC PROBLEMS WITH NONMONOTONE DISCONTINUITIES
}

\author{
by DIMITRIOS A. KANDILAKIS and NIKOLAOS S. PAPAGEORGIOU
}

(Received 9th January 1996)

\begin{abstract}
In this paper we consider a nonlinear periodic parabolic boundary value problem with a discontinuous nonmonotone nonlinearity. Using a lifting result for operators of type $\left(S_{+}\right)$, a general surjectivity theorem for operators of monotone type and an auxiliary problem defined by truncation and penalization we prove the existence of a solution in the order interval formed by an upper and lower solution. Moreover we show that the set of all such solutions is compact in $L^{p}\left(T, W_{0}^{1, p}(Z)\right)$.
\end{abstract}

1991 Mathematics subject classification: 35K55.

\section{Introduction}

Let $T=[0, b]$ and $Z \subseteq R^{N}$ a bounded domain with a $C^{1}$-boundary $\Gamma$. In this paper we study the following nonlinear periodic parabolic boundary value problem:

$$
\left\{\begin{array}{c}
\frac{\partial x}{\partial t}-\sum_{k=1}^{N} D_{k} a_{k}(t, z, x, D x)=f(x, D x) \text { on } T \times Z \\
x(0, z)=x(b, z) \text { a.e. on } Z,\left.x\right|_{T \times \Gamma}=0
\end{array}\right\}
$$

Here $D_{k}=\frac{\partial}{\partial z_{k}}, k \in\{1,2, \ldots N\}, D=\left(D_{k}\right)_{k=1}^{N}=\operatorname{grad}$ and $f: R \times R^{N} \rightarrow R$ is a locally bounded, measurable but not necessarily continuous function. In the absence of continuity hypotheses on $f(x, y)$ we know that even the corresponding initial boundary value problem need not have a solution. In this case it is a good idea to consider instead a multivalued version of (1) for which an adequate existence theory can be established. Roughly speaking the appropriate multivalued variant of the original problem can be obtained by filling in the gaps at the discontinuity points of $f(.$, .). This approach was first considered by Filippov [8] in the context of systems of ordinary differential equations. Later Rauch [13] considered semilinear elliptic problems and his work was extended further by Stuart [14] (who established the existence of maximal and minimal solutions within the interval determined by a lower and an upper solution) and by Chang [3] (whose approach is based on the critical point theory for nondifferential functionals). Since then most of the works deal with the stationary (elliptic) problem, while the study of the corresponding dynamic (parabolic) problem is lagging behind. Only recently some special classes of semilinear problems were considered by Carl and Heikkila [2], Feireisl [6] and Feireisl and Norbury [7]. In this paper we go 


\section{DIMITRIOS A. KANDILAKIS AND NIKOLAOS S. PAPAGEORGIOU}

beyond these works and consider a fully nonlinear problem with the discontinuous term depending also on the gradient $D x(.$, .) of $x$.

The multivalued version of (1) that we will be studying is defined in the following way. We set $f(x, y)=\varliminf_{\left(x^{\prime}, y\right) \rightarrow(x, y)} f\left(x^{\prime}, y^{\prime}\right)$ and $\bar{f}(x, y)=\overline{\lim }_{\left(x^{\prime}, y\right) \rightarrow(x, y)} f\left(x^{\prime}, y^{\prime}\right)$ and then define the multivalued (set-valued function) $(x, y) \rightarrow F(x, y)=[f(x, y), f(x, y)]$. Then we consider the following multivalued variant of problem (1)

$$
\left\{\begin{array}{c}
\frac{\partial x}{\partial t}-\sum_{k=1}^{N} D_{k} a_{k}(t, z, x, D x) \in F(x, D x) \text { on } T \times Z \\
x(0, z)=x(b, z) \text { a.e. on } Z,\left.x\right|_{T \times \Gamma}=0
\end{array}\right\}
$$

It is problem (2) that we will study. Our approach is based on some properties of operators of monotone type and on the method of upper and lower solutions. Our existence theorem extends the result of Deuel and Hess [4] who assume that the upper and lower solutions belong in $L^{\infty}(T \times Z), f(x, y)$ is continuous and the growth condition on $f(.,$.$) is more$ restrictive than ours. In addition the approach of Deuel and Hess [4] is different from ours and is based on an auxiliary variational inequality which the authors solve (see $[4, \mathrm{p}$. 95]). Instead here we rely on a general surjectivity result for a class of nonlinear pseudomonotone operators. In order to be able to use that theorem we also prove a result for operators of type $\left(S_{+}\right)$which is actually of independent interest (see Proposition 3.6).

\section{Preliminaries}

In this section we introduce some auxiliary material that we will need in the sequel and also fix our hypotheses concerning the data of problem (1).

Let $2 \leq p<\infty$ and let $W^{1, p}(Z)$ be the usual Sobolev space and $W^{1, p}(Z)^{*}$ its dual. The spaces $W^{1, p}(Z) \subseteq L^{2}(Z) \subseteq W^{1, p}(Z)^{*}$ form an evolution triple with all the embeddings being continuous, dense and compact (see Zeidler [16, Section 23.4 p. 416]). Also by $W_{0}^{1, p}(Z)$ we denote the subspace of $W^{1, p}(Z)$ whose elements have zero trace. As usual the dual of $W_{0}^{1, p}(Z)$ is denoted by $W^{-1, q}(Z)$ where $\frac{1}{p}+\frac{1}{q}=1$. Then is $W_{0}^{1, p}(Z) \subseteq L^{2}(Z) \subseteq W^{-1, q}(Z)$ also an evolution triple with all the embeddings being again continuous, dense and compact. We introduce the following function spaces:

$$
\begin{aligned}
\hat{W}_{p q}(T) & =\left\{f \in L^{p}\left(T, W^{1, p}(Z)\right): \frac{\partial f}{\partial t} \in L^{q}\left(T, W^{1, p}(Z)^{*}\right)\right\} \\
\text { and } W_{p q}(T) & =\left\{f \in L^{p}\left(T, W_{0}^{1, p}(Z)\right): \frac{\partial f}{\partial t} \in L^{q}\left(T, W^{-1, q}(Z)\right)\right\}
\end{aligned}
$$

In these definitions the derivatives $\frac{\partial f}{\partial t}$ are defined in the sense of vector-valued distributions. Both spaces equipped with the obvious norm $\|f\|_{p q}=\|f\|_{p}+\left\|\frac{\partial}{\partial t}\right\|_{q}$ become separable, reflexive Banach spaces. Moreover both $\hat{W}_{p q}(T)$ and $W_{p q}(T)$ embed continuously in $C\left(T, L^{2}(Z)\right.$ ) and compactly in $L^{p}(T \times Z)$ (see Lions [11, Theorem 5.1] or Zeidler [16, Proposition 23.23 p. 422 and p. 450]). 
Now let us introduce our hypotheses on the functions $a_{k}(t, z, x, y), k \in\{1,2, \ldots N\}$, involved in the definition of the partial differential operator in problem (1).

H(a): $\quad a_{k}: T \times Z \times R \times R^{N} \rightarrow R, k \in\{1,2, \ldots N\}$, are functions such that:

(i) $(t, z) \rightarrow a_{k}(t, z, x, y)$ is measurable;

(ii) $(x, y) \rightarrow a_{k}(t, z, x, y)$ is continuous;

(iii) $\sum_{k=1}^{N}\left(a_{k}(t, z, x, y)-a_{k}\left(t, z, x, y^{\prime}\right)\right)\left(y_{k}-y_{k}^{\prime}\right)>0$ for almost all $(t, z) \in T \times Z$, all $x \in R$ and all $y, y^{\prime} \in R^{N}, y \neq y^{\prime}$; and

(iv) $\sum_{k=1}^{N} a_{k}(t, z, x, y) y_{k} \geq c_{1}\|y\|^{p}-\beta_{1}(t, z)$ a.e. on $T \times Z$ with $\beta_{1} \in L^{1}(T \times Z), c_{1}>0$;

(v) $\left|a_{k}(t, z, x, y)\right| \leq \beta_{2}(t, z)+c_{2}\left(|x|^{p-1}+\|y\|^{p-1}\right)$ a.e. on $T \times Z$ with $\beta_{2} \in L^{q}(T \times Z)$, $c_{2}>0$ (here as before $2 \leq p<\infty$ and $\frac{1}{p}+\frac{1}{q}=1$ ).

Because of hypothesis $H(a)$ we can define the semilinear Dirichlet form $a: L^{p}\left(T, W^{1, p}(Z)\right) \times L^{p}\left(T, W^{1, p}(Z)\right) \rightarrow R$ by

$$
a(x, y)=\int_{0}^{b} \int_{z} \sum_{k=1}^{N} a_{k}(t, z, x, D x) D_{k} y(t, z) d z d t
$$

In what follows by $((.,)$.$) we will denote the duality brackets for the pairs$ $\left(L^{p}\left(T, W^{1, p}(Z)\right), L^{q}\left(T, W^{1, p}(Z)^{*}\right)\right)$ and $\left(L^{p}\left(T, W_{0}^{1, p}(Z)\right), L^{q}\left(T, W^{-1, q}(Z)\right)\right)$. Recall that if $X$ is a reflexive Banach space (or more generally if $X^{*}$ has the Radon-Nikodym property) and $1 \leq p<\infty$, then $L^{p}(T, X)^{*}=L^{q}\left(T, X^{*}\right)$ with $\frac{1}{p}+\frac{1}{q}=1$ (see Diestel and Uhl [5, Theorem 1]). (2) if

Definition 2.1. A function $\varphi \in \hat{W}_{p q}(T)$ is said to be an upper solution of problem

$$
\left(\left(\frac{\partial \varphi}{\partial t}, u\right)\right)+a(\varphi, u) \geq \int_{0}^{b} \int_{z} \bar{f}(\varphi(t, z), D \varphi(t, z)) u(t, z) d z d t
$$

for all $u \in L^{p}\left(T, W_{0}^{1, p}(Z)\right) \cap L^{p}(T \times Z)_{+}, \varphi(0, z) \geq \varphi(b, z)$ a.e. on $Z$ and $\left.\varphi\right|_{\tau \times \Gamma} \geq 0$. Similarly a function $\psi \in \hat{W}_{p q}(T)$ is said to be a lower solution of problem (2) if the above inequalities are reversed and $\bar{f}$ is replaced by $\underline{f}$.

We make the following hypothesis concerning the existence of lower and upper solutions of problem (1):

$\mathbf{H}_{0}$ : there exist an upper solution $\varphi \in \hat{W}_{p q}(T)$ and a lower solution $\psi \in \hat{W}_{p q}(T)$ such that $\psi(t, z) \leq \varphi(t, z)$ a.e. on $T \times Z$. 
Our hypothesis on the discontinuous term $f(.,$.$) is the following:$

H(f): $f: R \times R^{N} \rightarrow R$ is a measurable function such that for almost all $(t, z) \in T \times Z$ and all $v \in[\psi(t, z), \varphi(t, z)],|f(v, y)| \leq \beta(t, z)+c\|y\|^{p-1}$ a.e. on $T \times Z$ with $\beta_{3} \in L^{q}(T \times Z)$ and $c_{3}>0$.

Now we introduce the notion of a (weak) solution for problem (2).

Definition 2.2. A function $x \in W_{p q}(T)$ is said to be a solution of (2), if there exists a function $g \in L^{q}(T \times Z)$ such that $g(t, z) \in F(x(t, z), D x(t, z))$ a.e. on $T \times Z$ and

$$
\left(\left(\frac{\partial x}{\partial t}, u\right)\right)+a(x, u)=\int_{0}^{b} \int_{z} g(t, z) u(t, z) d z d t
$$

for all $u \in L^{p}\left(T, W_{0}^{1, p}(Z)\right), x(0, z)=x(b, z)$ a.e. on $Z$ and $\left.x\right|_{T \times \Gamma}=0$.

We will establish the existence of solutions for problem (2) by solving an auxiliary periodic boundary value problem which is defined through truncation and penalization.

\section{Auxiliary results}

First we introduce the truncation operator. So given $x \in L^{p}\left(T, W^{1, p}(Z)\right)$ we define its truncation $\tau(x)(.,$.$) as follows:$

$$
\tau(x)(t, z)= \begin{cases}\varphi(t, z) & \text { if } \varphi(t, z) \leq x(t, z) \\ x(t, z) & \text { if } \psi(t, z) \leq x(t, z) \leq \varphi(t, z) \\ \psi(t, z) & \text { if } x(t, z) \leq \psi(t, z)\end{cases}
$$

Proposition 3.1. $\tau: L^{p}\left(T, W^{1, p}(Z)\right) \rightarrow L^{p}\left(T, W^{1, p}(Z)\right)$ is continuous.

Proof. From Lemma 7.6, p. 145 of Gilbarg and Trudinger [9] we know that given $x \in L^{p}\left(T, W^{1, p}(Z)\right)$, for almost all $t \in T \tau(x)(t,.) \in W^{1, p}(Z)$ and also

$$
D \tau(x)(t, z)= \begin{cases}D \varphi(t, z) & \text { if } \varphi(t, z) \leq x(t, z) \\ D x(t, z) & \text { if } \psi(t, z) \leq x(t, z) \leq \varphi(t, z) \\ D \psi(t, z) & \text { if } x(t, z) \leq \psi(t, z)\end{cases}
$$

Hence, $\tau(x)(.,.) \in L^{p}\left(T, W^{1, p}(Z)\right)$. Now let $x_{n} \rightarrow x$ in $L^{p}\left(T, W^{1, p}(Z)\right)$ as $n \rightarrow \infty$. Then by passing to a subsequence if necessary we may assume that $x_{n}(t, z) \rightarrow x(t, z)$ and $D x_{n}(t, z) \rightarrow D x(t, z)$ a.e. on $T \times Z$ as $n \rightarrow \infty$ for every $k \in\{1,2, \ldots N\}$. In addition by 
virtue of Theorem 2.8.1, p. 74 of [10] we can find functions $\theta, \theta_{k} \in L^{p}(T \times Z)$, $k \in\{1,2, \ldots N\}$ such that $\left|x_{n}(t, z)\right| \leq \theta(t, z)$ and $\left|D_{k} x_{n}(t, z)\right| \leq \theta_{k}(t, z)$ a.e. on $T \times Z$. Observe that $\left|\tau\left(x_{n}\right)(t, z)\right| \leq \max \{\theta(t, z),|\varphi(t, z)|,|\psi(t, z)|\}$ a.e. on $T \times Z$ and $\left|D_{k} \tau\left(x_{n}\right)(t, z)\right| \leq \max \left\{\theta_{k}(t, z), \quad\left|D_{k} \varphi(t, z)\right|, \quad\left|D_{k} \psi(t, z)\right|\right\} \quad$ a.e. on $T \times Z$ for all $k \in\{1,2, \ldots N\}$ and all $n \geq 1$. Thus the dominated convergence theorem implies that $x_{n} \rightarrow x$ in $L^{p}(T \times Z)$ and $D x_{n} \rightarrow D x$ in $L^{p}\left(T \times Z, R^{N}\right)$ as $n \rightarrow \infty$. Therefore we can conclude that $\tau\left(x_{n}\right) \rightarrow \tau(x)$ in $L^{p}\left(T, W^{1, p}(Z)\right)$ as $n \rightarrow \infty$, which proves the continuity of the truncation map $\tau($.).

Next we introduce the penalty function $u: T \times Z \times R \rightarrow R$ defined by

$$
u(t, z, x)= \begin{cases}(x-\varphi(t, z))^{p-1} & \text { if } \varphi(t, z) \leq x \\ 0 & \text { if } \psi(t, z) \leq x \leq \varphi(t, z) \\ -(\psi(t, z)-x)^{p-1} & \text { if } x \leq \psi(t, z)\end{cases}
$$

This definition and a routine calculation give us the following properties of the penalty function $u(t, z, x)$.

Proposition 3.2. $u: T \times Z \times R \rightarrow R$ is a Caratheodory function (i.e. measurable in $(t, z)$ and continuous in $x),|u(t, z, x)| \leq \beta_{4}(t, z)+c_{4}|x|^{p-1}$ a.e. on $T \times Z$ with $\beta_{4} \in L^{q}(T \times Z), c_{4}>0$, and $\int_{0}^{b} \int_{Z} u(t, z, x(t, z)) x(t, z) d z d t \geq c_{5}\|x\|_{L(T \times Z)}-c_{6}\|x\|_{L(T \times Z)}^{p-1}$ for some $c_{5}, c_{6}>0$ and for all $x \in L^{p}(T \times Z)$.

Next let $X=W_{0}^{1, p}(Z)$. By virtue of hypothesis $H(a)$ we can define the operator $A: T \times X \rightarrow X^{*}$ by

$$
\langle A(t, x), y\rangle=\sum_{k=1}^{N} \int_{Z} a_{k}(t, z, x, D x) D_{k} y(z) d z
$$

for all $y \in X$. Here by $\langle.,$.$\rangle we denote the duality brackets for the pair \left(W_{0}^{1, p}(Z)\right.$, $\left.W^{-1, q}(Z)\right)$. In the next proposition we establish a useful property of $x \rightarrow A(t, x)$. First a definition (see for example Zeidler [16, p. 583]).

Definition 3.1. If $Y$ is a reflexive Banach space and $A: Y \rightarrow Y^{*}$ an operator, we say that $A($.$) is of type \left(\mathrm{S}_{+}\right)$if $x_{n} \stackrel{w}{\rightarrow} x$ in $Y$ as $n \rightarrow \infty$ and $\lim \left\langle A\left(x_{n}\right)-A(x), x_{n}-x\right\rangle \leq 0$ imply that $x_{n} \rightarrow x$ in $Y$ as $n \rightarrow \infty$ (as before by $\langle.,$.$\rangle we denote the duality brackets$ for the pair $\left.\left(Y, Y^{*}\right)\right)$.

Remark 3.1. A uniformly monotone operator is of type $\left(S_{+}\right)$(see Zeidler [16, p. 584]).

Proposition 3.3. If hypothesis $H(a)$ holds and $A: T \times X \rightarrow X^{*}$ is defined as above, then $t \rightarrow A(t, x)$ is measurable and $x \rightarrow A(t, x)$ is demicontinuous and of type $\left(S_{+}\right)$. 
Proof. By Fubini's theorem for every $y \in X t \rightarrow\langle A(t, x), y\rangle$ is measurable. So $t \rightarrow A(t, x)$ is weakly measurable and since $X^{*}=W^{-1, q}(Z)$ is separable, from the Pettis measurability theorem (see Diestel and Uhl [5, Theorem 2, p. 42]) we conclude that $t \rightarrow A(t, x)$ is measurable. Next let $x_{n} \rightarrow x$ in $X=W_{0}^{1, p}(Z)$ as $n \rightarrow \infty$. Then by passing to a subsequence if necessary we may assume that $x_{n}(z) \rightarrow x(z)$ and $D x_{n}(z) \rightarrow D x(z)$ a.e. on $Z$ as $n \rightarrow \infty$. By virtue of hypothesis $H(a)(i i)$ $a_{k}\left(t, z, x_{n}(z), D x_{n}(z)\right) \rightarrow a_{k}(t, z, x(z), D x(z))$ a.e. on $Z$ as $n \rightarrow \infty$ for all $k \in\{1,2, \ldots N\}$ and so by the dominated convergence theorem (see hypothesis $H(a)(v)$ ) we infer that for all $y \in X=W_{0}^{1, p}(Z)$

$$
\begin{aligned}
\left\langle A\left(t, x_{n}\right), y\right\rangle & =\sum_{k=1}^{N} \int_{z} a_{k}\left(t, z, x_{n}(z), D x_{n}(z)\right) D_{k} y(z) d z \\
& \rightarrow \sum_{k=1}^{N} \int_{z} a_{k}(t, z, x(z), D x(z)) D_{k} y(z) d z=\langle A(t, x), y\rangle
\end{aligned}
$$

Since $y \in Y$ was arbitrary, we conclude that $A\left(t, x_{n}\right) \stackrel{w}{\rightarrow} A(t, x)$ in $X^{*}$ as $n \rightarrow \infty$ and this proves the demicontinuity of $A(t,$.$) .$

Finally we will show that $A(t,$.$) is of type \left(\mathrm{S}_{+}\right)$. To this end let $x_{n} \stackrel{w}{\rightarrow} x$ in $X$ as $n \rightarrow \infty$ and assume that $\overline{\lim }\left(A\left(t, x_{n}\right)-A(t, x), x_{n}-x\right) \leq 0$. By virtue of hypothesis $\mathrm{H}$ (a)(iii) $\left\langle A\left(t, x_{n}\right)-A(t, x), x_{n}-x\right\rangle \geq 0$ for all $n \geq 1$. So $\left\langle A\left(t, x_{n}\right)-A(t, x), x_{n}-x\right\rangle \rightarrow 0$ as $n \rightarrow \infty$. From [10, Theorem 2.8.1], we know that by passing to a subsequence if necessary, we may assume that

$$
\sum_{k=1}^{N}\left(a_{k}\left(t, z, x_{n}, D x_{n}\right)-a_{k}(t, z, x, D x)\right) D_{k}\left(x_{n}-x\right)(z) \leq h_{1}(z)
$$

for all $z \in Z \backslash N_{1}, \lambda\left(N_{1}\right)=0$, with $h_{1} \in L^{1}(Z)$ and $\lambda($.$) being the Lebesgue measure on$ $Z$. Using hypotheses $H(a)$ (iv) and (v) we see that for every $z \in Z \backslash N_{1}$ and $n \geq 1$

$$
\begin{aligned}
h_{1}(z) & \geq \sum_{k=1}^{N}\left(a_{k}\left(t, z, x_{n}, D x_{n}\right)-a_{k}(t, z, x, D x)\right) D_{k}\left(x_{n}-x\right)(z) \\
& \geq c_{1}\left(\left\|D x_{n}(z)\right\|^{p}+\|D x(z)\|^{p}\right)-2 \beta_{1}(t, z) \\
& -\sum_{k=1}^{N}\left|D_{k} x(z)\right|\left(\beta_{2}(t, z)+c_{2}\left(\left|x_{n}(z)\right|^{p-1}+\left|D_{k} x_{n}(z)\right|^{p-1}\right)\right) \\
& -\sum_{k=1}^{N}\left|D_{k} x_{n}(z)\right|\left(\beta_{2}(t, z)+c_{2}\left(|x(z)|^{p-1}+\left|D_{k} x(z)\right|^{p-1}\right)\right) .
\end{aligned}
$$

Since by hypothesis $x_{n} \stackrel{w}{\rightarrow} x$ in $X$ and $X=W_{0}^{1, p}(Z)$ embeds compactly in $L^{p}(Z)$, we deduce that $x_{n} \rightarrow x$ in $L^{p}(Z)$ as $n \rightarrow \infty$. Then by passing to a subsequence if necessary, 
we may assume that $x_{n}(z) \rightarrow x(z)$ for all $z \in Z \backslash N_{2}, \lambda\left(N_{2}\right)=0$ and $\left|x_{n}(z)\right| \leq h_{2}(z)$ for all $z \in Z \backslash N_{2}$ with $h_{2} \in L^{\prime}(Z)$. Using this fact in (3) we infer that for all $z \in Z \backslash N$, $N=N_{1} \cup N_{2}, \lambda(N)=0$, the sequence $\left\{\left\|D x_{n}(z)\right\|\right\}_{n \geq 1}$ is bounded. So for fixed $z \in Z \backslash N$ we can find a subsequence $\left\{x_{m}(z)\right\}_{m \geq 1}$ of $\left\{x_{n}(z)\right\}_{n \geq 1}$ such that $x_{m}(z) \rightarrow x(z)$ and $D_{k} x_{m}(z) \rightarrow y_{k}(z)$ as $m \rightarrow \infty$. Hence passing to the limit as $m \rightarrow \infty$ we obtain for all $z \in Z \backslash N$

$$
\sum_{k=1}^{N}\left(a_{k}(t, z, x, y)-a_{k}(t, z, x, D x)\right)\left(y_{k}-D_{k} x\right)(z)=0
$$

and so $y_{k}(z)=D_{k} x(z)$ for all $k \in\{1,2, \ldots N\}$ (see hypothesis $H(a)$ (iii)). Since the limits $y_{k}(z)$ are uniquely determined, we deduce that $D x_{n}(z) \rightarrow D x(z)$ for all $z \in Z \backslash N$ as $n \rightarrow \infty$. Moreover from (3) it follows that

$$
\begin{aligned}
\left\|D x_{n}(z)\right\|^{p} & \leq h_{1}(z)+c_{1}\|D x(z)\|^{p}+2 \beta_{1}(t, z) \\
& +\sum_{k=1}^{N}\left|D_{k} x(z)\right|\left(\beta_{2}(t, z)+c_{2}\left(\left|x_{n}(z)\right|^{p-1}+\left|D_{k} x_{n}(z)\right|^{p-1}\right)\right) \\
& +\sum_{k=1}^{N}\left|D_{k} x_{n}(z)\right|\left(\beta_{2}(t, z)+c_{2}\left(|x(z)|^{p-1}+\left|D_{k} x(z)\right|^{p-1}\right)\right)
\end{aligned}
$$

for all $z \in Z \backslash N$. Note that for $C \subseteq Z$ measurable

$$
\begin{aligned}
& \sum_{k=1}^{N} \int_{C}\left|D_{k} x(z)\right|\left(\beta_{2}(t, z)+c_{2}\left(\left|x_{n}(z)\right|^{p-1}+\left|D_{k} x_{n}(z)\right|^{p-1}\right)\right) d z \\
& \quad \leq \sum_{k=1}^{N}\left\|\chi_{C} D_{k} x\right\|_{p}\left\|\beta_{2}(t, .)+c_{2}\left(\left|x_{n}(.)\right|^{p-1}+\left|D_{k} x_{n}(.)\right|^{p-1}\right)\right\|_{q} \\
& \quad \leq c_{4}\left(\sum_{k=1}^{N}\left\|\chi_{C} D_{k} x_{n}\right\|_{p}^{p}\right)^{\frac{1}{p}}\left(\sum_{k=1}^{N}\left\|\beta_{2}(t, .)\right\|_{q}^{q}\right. \\
& \left.\quad+c_{2}\left\|x_{n}(.)\right\|_{p}^{p}+c_{2}\left\|D_{k} x_{n}\right\|_{p}^{p}\right)^{\frac{1}{q}} \text { for some } c_{4}>0 \\
& \quad \leq c_{5}\left(\sum_{k=1}^{N} \int_{C}\left|D_{k} x_{n}(z)\right|^{p} d z\right)^{\frac{1}{p}} \text { for some } c_{5}>0 .
\end{aligned}
$$

Also 


$$
\begin{aligned}
& \sum_{k=1}^{N} \int_{C}\left|D_{k} x_{n}(z)\right|\left(\beta_{2}(t, z)+c_{2}\left(|x(z)|^{p-1}+\left|D_{k} x(z)\right|^{p-1}\right)\right) d z \\
& \quad \leq \sum_{k=1}^{N}\left\|\chi_{c}\left(\beta_{2}(t, .)+c_{2}\left(|x(.)|^{p-1}+\left|D_{k} x(.)\right|^{p-1}\right)\right)\right\|_{q}\left\|D_{k} x_{n}\right\|_{p} \\
& \quad \leq c_{6} \sum_{k=1}^{N} \int_{C}\left(\beta_{2}(t, z)+c_{2}\left(|x(z)|^{p-1}+\left|D_{k} x(z)\right|^{p-1}\right)\right) d z
\end{aligned}
$$

for some $c_{6}>0$. From (4), (5) and (6) we deduce at once that $\left\{\left\|D x_{n}(.)\right\|^{p}\right\}_{n \geq 1}$ is uniformly integrable. Hence from the generalized dominated convergence theorem (see for example Ash [1, Theorem 7.5.2]), we infer that $D x_{n} \rightarrow D x$ in $L^{p}\left(Z, R^{N}\right)$ as $n \rightarrow \infty$. Therefore $x_{n} \rightarrow x$ in $X=W_{0}^{1, p}(Z)$ as $n \rightarrow \infty$ and so $A(t,$.$) is of type \left(S_{+}\right)$.

Our proof of the main existence theorem (see Section 4) will use an auxiliary periodic problem and a general surjectivity result for the sum of two operators of monotone type. Although the result is known (see for example Lions [11, Theorem 1.2] or B-A. Ton [15, Corollary 1]), nevertheless for the convenience of the reader we recall it here. We start with a definition:

Definition 3.2. Suppose that $Y$ is a reflexive Banach space, $L: D(L) \subseteq Y \rightarrow Y^{*}$ is a linear densely defined maximal monotone operator and $V: Y \rightarrow 2^{r^{*}} \backslash\{\emptyset\}$ a multivalued operator with weakly compact and convex values. We will say that $V($.$) is pseudomono-$ tone with respect to $D(L)$ if for $\left\{y_{n}\right\}_{n \geq 1} \subseteq D(L)$ with $y_{n} \stackrel{w}{\rightarrow} y$ in $Y$ and $L\left(y_{n}\right) \stackrel{w}{\rightarrow} L(y)$ in $Y^{*}$ as $n \rightarrow \infty$ and for $y_{n}^{*} \in V\left(y_{n}\right), n \geq 1$, satisfying $y_{n}^{*} \stackrel{w}{\rightarrow} y^{*}$ in $Y^{*}$ as $n \rightarrow \infty$ and $\varlimsup \lim \left(y_{n}^{*}, y\right) \leq\left(y^{*}, y\right)$, we can deduce that $y^{*} \in V(y)$ and $\left(y_{n}^{*}, y_{n}\right) \rightarrow\left(y^{*}, y\right)$ as $n \rightarrow \infty$.

In a similar way we can define the notion of an operator of type $\left(S_{+}\right)$with respect to $D(L)$ :

Definition 3.3. Let $Y$ and $L: D(L) \subseteq Y \rightarrow Y^{*}$ be as in the previous definition. An operator $V: Y \rightarrow Y^{*}$ is said to be of type $\left(\mathrm{S}_{+}\right)$with respect to $D(L)$ if for $\left\{y_{n}\right\}_{n \geq 1} \subseteq D(L)$ with $y_{n} \stackrel{w}{\rightarrow} y$ in $Y$ and $L\left(y_{n}\right) \stackrel{w}{\rightarrow} L(y)$ in $Y^{*}$ as $n \rightarrow \infty$ for which $\overline{\lim }\left(V\left(y_{n}\right), y_{n}-y\right) \leq 0$, we obtain $y_{n} \rightarrow y$ in $Y$ as $n \rightarrow \infty$.

The surjectivity result that will give us a solution for the auxiliary problem is the following:

Proposition 3.4. If $Y$ is a reflexive Banach space, $L: D(L) \subseteq Y \rightarrow Y^{*}$ is a linear densely defined maximal monotone operator and $V: Y \rightarrow 2^{Y^{*}} \backslash\{\emptyset\}$ is a multivalued map with weakly compact and convex values, which is bounded (i.e. maps bounded sets to bounded sets), pseudomonotone with respect to $D(L)$, sequentially closed in $Y \times Y_{w}^{*}$ (here $Y_{w}^{*}$ denotes the Banach space $Y^{*}$ furnished with the weak topology) and coercive (i.e. 
$\inf \left\{\frac{\left(y^{*}, y\right)}{|y|}: y^{*} \in V(y)\right\} \rightarrow \infty$ as $\left.\|y\| \rightarrow \infty\right)$, then $R(L+V)=Y^{*}$ (i.e. the operator $(L+V)($.$) is surjective).$

Now we recall from the general theory of maximal monotone operators (see for example Zeidler [16, Theorem 32.I]) that $L: D(L) \subseteq Y \rightarrow Y^{*}$ is a linear densely defined maximal monotone operator iff $L($.$) is closed and monotone and L^{*}($.$) is also$ monotone.

Using this fact we can easily establish the next proposition concerning the operator $L($.) of our problem.

Proposition 3.5. If $L: D(L) \subseteq L^{p}\left(T, W_{0}^{1, p}(Z)\right) \rightarrow L^{q}\left(T, W^{-1, q}(Z)\right)$ is defined by $L(x)=\dot{x}$ (as before the time derivative is defined in the sense of vector valued distributions) with $x \in D(L)=\left\{y \in L^{p}\left(T, W_{0}^{1, p}(Z)\right): \dot{y} \in L^{q}\left(T, W^{-1, q}(Z)\right), \quad y(0)=y(b)\right\} \subseteq W_{p q}(T)$, then $L($.$) is a densely defined, linear and maximal monotone operator.$

Proof. Observe that $L($.$) is linear, monotone and densely defined. The density of$ the domain $D(L)$ of $L($.$) in L^{P}\left(T, W_{0}^{1, p}(Z)\right)$ follows from the fact that the functions $y \in C^{\infty}\left(T, W_{0}^{1, p}(Z)\right)$ such that $y(0)=y(b)$ are dense in $L^{p}\left(T, W_{0}^{1, p}(Z)\right)$. This last statement is proved easily by means of homogenizing operators (see for example Zeidler [16] or Zhikov, Kozlov and Oleinik [17]). Also $L^{*}: D\left(L^{*}\right) \subseteq L^{p}\left(T, W_{0}^{1, p}(Z)\right) \rightarrow$ $L^{q}\left(T, W^{-1, q}(Z)\right)$ is defined by $L^{*}(y)=-\dot{y}$ for all $y \in D\left(L^{*}\right)=D(L)$. Hence $L^{*}$ is monotone too and so we conclude that $L$ is maximal monotone.

Now let $\hat{A}: L^{p}(T, X) \rightarrow L^{q}\left(T, X^{*}\right)$ be the Nemitsky (superposition) operator corresponding to $A(t, x)$; i.e. $\hat{A}(x)()=.A(., x()$.$) . From Proposition 3.3$ we know that for every $y \in X$ the function $(t, v) \rightarrow\langle A(t, v), y\rangle$ is measurable in $t$, continuous in $v$ (i.e. a Caratheodory function) hence it is jointly measurable. So if $x \in L^{p}(T, X)$ then $t \rightarrow\langle A(t, x(t)), y\rangle$ is measurable on $T$. Because $y \in X$ was arbitrary we infer that $t \rightarrow A(t, x(t))$ is weakly measurable from $T$ into $X^{*}=W^{-1, q}(Z)$ and since the latter is separable reflexive, as before from the Pettis measurability theorem, we conclude that $t \rightarrow A(t, x(t))$ is measurable. Then hypothesis $\mathrm{H}(\mathrm{a})(\mathrm{v})$ tells us that $A(., x())=$. $\hat{A}(x)(.) \in L^{q}\left(T, X^{*}\right)$.

In the next proposition we show that the property that $A(t,$.$) is of type \left(\mathrm{S}_{+}\right)$(see Proposition 3.3.) can be lifted to $\hat{A}($.$) in the sense that \hat{A}($.$) is of type \left(S_{+}\right)$with respect to $D(L)$.

Proposition 3.6. If hypothesis $\mathrm{H}(\mathrm{a})$ holds and $\hat{A}: L^{P}(T, X) \rightarrow L^{q}\left(T, X^{*}\right)$ is defined as above, then $\hat{A}($.$) is of type \left(\mathrm{S}_{+}\right)$with respect to $D(L)$.

Proof. Let $\left\{x_{n}\right\}_{n \geq 1} \subseteq D(L)$ and assume that $x_{n} \stackrel{w}{\rightarrow} x$ in $W_{p q}(T)$ as $n \rightarrow \infty$ (i.e. $x_{n} \stackrel{w}{\rightarrow} x$ in $L^{P}(T, X)$ and $\dot{x}_{n} \stackrel{w}{\rightarrow} \dot{x}$ in $L^{q}\left(T, X^{*}\right)$ as $\left.n \rightarrow \infty\right)$ and that $\overline{\lim }\left(\left(\hat{A}\left(x_{n}\right), x_{n}-x\right)\right) \leq 0$ (recall that by $((.,)$.$\left.) we denote the duality brackets for the pair \left(L^{p}(T, X), L^{q}\left(T, X^{*}\right)\right)\right)$. Put $\xi_{n}(t)=\left(A\left(t, x_{n}(t)\right), x_{n}(t)-x(t)\right)$. Since $W_{p q}(T)$ embeds continuously in $C\left(T, L^{2}(Z)\right)$, we 


\section{DIMITRIOS A. KANDILAKIS AND NIKOLAOS S. PAPAGEORGIOU}

deduce that $x_{n} \stackrel{w}{\rightarrow} x$ in $C\left(T, L^{2}(Z)\right)$ as $n \rightarrow \infty$ and so for every $t \in T x_{n}(t) \stackrel{w}{\rightarrow} x(t)$ in $L^{2}(Z)$ as $n \rightarrow \infty$. On the other hand let $N \subseteq T$ be the exceptional Lebesgue-null set outside of which we have $\langle A(t, x), x\rangle \geq \hat{c}_{1}\|x\|_{X}^{p}-\hat{\beta}_{1}(t)$ for some $\hat{c}_{1}>0$ and $\hat{\beta}_{1} \in L^{1}(T)$ and $\|A(t, x)\|_{X^{*}} \leq \hat{\beta}_{2}(t)+\hat{c}_{2}\|x\|_{X}^{p-1}$ for some $\hat{\beta}_{2} \in L^{q}(T)$ and $\hat{c}_{2}>0$ (see hypotheses $\mathrm{H}(\mathrm{a})(\mathrm{iv})$ and (v)). Then for $t \in T \backslash N$ we can write

$$
\xi_{n}(t) \geq \varphi_{n}(t)=\hat{c}_{1}\|x\|_{X}^{p}-\hat{\beta}_{1}(t)-\left(\hat{\beta}_{2}(t)+\hat{c}_{2}\|x\|_{X}^{p-1}\right)\|x(t)\|_{X}
$$

If $C=\left\{t \in T:\left\lfloor\underline{\lim } \xi_{n}(t)<0\right\}\right.$ (which is measurable) and $\mu(C)>0 \quad(\mu($.$) being the$ Lebesgue measure on $T$ ), then from (7) above we deduce that for $t \in C \cap(T \backslash N)$ $\left\{x_{n}(t)\right\}_{n \geq 1}$ is bounded in $X=W_{0}^{1, p}(Z)$. Since $X$ is reflexive and $x_{n}(t) \stackrel{\text { m }}{\rightarrow} x(t)$ in $L^{2}(Z)$ as $n \rightarrow \infty$, we infer that $x_{n}(t) \stackrel{W}{\rightarrow} x(t)$ in $X$ as $n \rightarrow \infty$. Fix $t \in C \cap(T \backslash N)$ and choose a suitable subsequence so that $\underline{\lim } \xi_{n}(t)=\lim \xi_{m_{k}}(t)$. Then by virtue of the fact that $A(t,$. is of type $\left(\mathrm{S}_{+}\right)$(see Proposition 3.3) we obtain that $x_{n_{k}}(t) \rightarrow x(t)$ in $X$ as $k \rightarrow \infty$ and so $\xi_{n_{k}}(t)=\left\langle A\left(t, x_{n_{k}}(t)\right), x_{n_{k}}(t)-x(t)\right\rangle \rightarrow 0$ as $k \rightarrow \infty$, which contradicts the definition of $C$. Therefore $\lim \xi_{n}(t) \geq 0$ a.e. on $T$. Then from Fatou's lemma it follows that

$$
\begin{aligned}
0 & \leq \int_{0}^{b} \underline{\lim } \xi_{n}(t) d t \leq \varliminf \int_{0}^{b} \xi_{n}(t) d t \\
& \leq \varlimsup \lim \int_{0}^{b} \xi_{n}(t) d t=\varlimsup\left(\hat{\lim }\left(\left(\hat{A}\left(x_{n}\right), x_{n}-x\right)\right) \leq 0\right.
\end{aligned}
$$

hence $\int_{0}^{b} \xi_{n}(t) d t \rightarrow 0$ as $n \rightarrow \infty$. Note that $\left|\xi_{n}(t)\right|=\xi_{n}^{+}(t)+\xi_{n}^{-}(t)=\xi_{n}(t)+2 \xi_{n}^{-}(t)$. Since $0 \leq \varliminf^{\prime} \xi_{n}(t)$ a.e. on $T$, we deduce that $\xi_{n}^{-}(t) \rightarrow 0$ a.e. on $T$ as $n \rightarrow \infty$. Recall that $\varphi_{n}(t) \leq \xi_{n}(t)$ a.e. on $T$ (see (7)) with $\left\{\varphi_{n}(.)\right\}_{n \geq 1}$ uniformly integrable. Then $0 \leq \xi_{n}^{-}(t) \leq \varphi_{n}^{-}(t)$ a.e. on $T$ and of course $\left\{\varphi_{n}^{-}(.)\right\}_{n \geq 1}$ is uniformly integrable too. So we can apply the extended dominated convergence theorem (see Ash [1, Theorem 7.5.2]) and obtain that $\int_{0}^{b} \xi_{n}^{-}(t) d t \rightarrow 0$ as $n \rightarrow \infty$. So finally we can write that $\int_{0}^{b}\left|\xi_{n}(t)\right| d t \rightarrow 0$ as $n \rightarrow \infty$ and so by passing to a subsequence if necessary we may also assume that $\xi_{n}(t) \rightarrow 0$ a.e. on $T$ as $n \rightarrow \infty$. Then because $A(t,$.$) is of type \left(\mathrm{S}_{+}\right)$(see Proposition 3.3) we obtain that $x_{n}(t) \rightarrow x(t)$ in $X$ a.e. on $T$ as $n \rightarrow \infty$. Also

$$
\begin{aligned}
& \left\|x_{n}(t)-x(t)\right\|_{X}^{p} \leq 2^{p-1}\left\|x_{n}(t)\right\|_{X}^{p}+2^{p-1}\|x(t)\|_{X}^{p} \\
& \quad \leq \frac{2^{p-1}}{\hat{c}_{1}}\left\langle A\left(t, x_{n}(t)\right), x_{n}(t)\right\rangle+\hat{\beta}_{1}(t)+2^{p-1}\|x(t)\|_{X}^{p} \\
& \quad=\frac{2^{p-1}}{\hat{c}_{1}}\left(\left\langle A\left(t, x_{n}(t)\right), x(t)\right\rangle+\xi_{n}(t)\right)+\hat{\beta}_{1}(t)+2^{p-1}\|x(t)\|_{X}^{p} \text { a.e. on } T
\end{aligned}
$$

from which it follows that $\left\{\left\|x_{n}(.)-x(.)\right\|_{x}^{p}\right\}_{n \geq 1}$ is uniformly integrable. Therefore a new application of the extended dominated convergence theorem gives us that $\left\|x_{n}-x\right\|_{L P(T, X)} \rightarrow 0$ as $n \rightarrow \infty$ so we conclude that $\hat{A}$ is of type $\left(S_{+}\right)$with respect to $D(L)$. 


\section{An existence theorem}

In this section using the auxiliary tools of Section 3 we will prove the existence of a solution $x \in W_{p q}(T)$ for problem (2) such that $\psi(t, z) \leq x(t, z) \leq \varphi(t, z)$ a.e. on $T \times Z$.

Theorem 4.1. If hypotheses $\mathrm{H}(\mathrm{a}), \mathrm{H}_{0}$ and $\mathrm{H}(\mathrm{f})$ hold, then problem (2) has a solution $x \in W_{p q}(T)$ such that $\psi(t, z) \leq x(t, z) \leq \varphi(t, z)$ a.e. on $T \times Z$. Moreover the set of all such solutions of $(2)$ is compact in $L^{p}\left(T, W_{0}^{1, p}(Z)\right)$.

Proof. Let $\tau: L^{p}\left(T, W^{1, p}(Z)\right) \rightarrow L^{p}\left(T, W^{1, p}(Z)\right)$ be the truncation at $\{\varphi, \psi\}$ map and $u: T \times Z \times R \rightarrow R$ the corresponding penalty function. We consider the following auxiliary periodic boundary value problem

$$
\left\{\begin{array}{c}
\frac{\partial x}{\partial t}-\sum_{k=1}^{N} D_{k} a_{k}(t, z, \tau(x), D x)+\lambda u(t, z, x(t, z)) \\
\in F(\tau(x)(t, z), D \tau(x)(t, z)) \text { on } T \times Z \\
x(0, z)=x(b, z) \text { a.e. on } Z,\left.x\right|_{T \times \Gamma}=0
\end{array}\right\}
$$

where $\lambda>0$ will be fixed in the process of the proof. Let $\hat{A}: L^{p}\left(T, W_{0}^{1, p}(Z)\right) \rightarrow$ $L^{q}\left(T, W^{-1, q}(Z)\right)$ be defined from (8) as in Section 3. Also define $U: L^{p}(T \times Z) \rightarrow$ $L^{q}(T \times Z)$ by $U(x)(t, z)=u(t, z, x(t, z))$ (the Nemitsky operator for the map $u(t, z, x)$ ) and $G: L^{p}\left(T, W_{0}^{1, p}(Z)\right) \rightarrow 2^{L^{q}(T \times Z)}$ by $G(x)=\left\{g \in L^{q}(T \times Z): g(t, z) \in \quad F(\tau(x)(t, z)\right.$, $D \tau(x)(t, z))$ a.e. on $T \times Z$ \}. Set $V(x)=\hat{A}(x)+\lambda U(x)-G(x)$.

Claim 1: $\quad V: L^{P}\left(T, W_{0}^{1, p}(Z)\right) \rightarrow 2^{L^{q}\left(T, W^{-1, q(Z))}\right.} \backslash\{\emptyset]$ is pseudomonotone with respect to $D(L)$.

It is clear that $V\left(\right.$.) has weakly compact and convex values. Next let $x_{n} \stackrel{W}{\rightarrow} x$ in $W_{p q}(T)$ as $n \rightarrow \infty, v_{n} \in V\left(x_{n}\right), n \geq 1$, with $v_{n} \stackrel{w}{\rightarrow} v$ in $L^{q}\left(T, W^{-1, q}(Z)\right)$ and assume that $\varlimsup \lim \left(\left(v_{n}, x_{n}-x\right)\right) \leq 0$. By definition $v_{n}=\hat{A}\left(x_{n}\right)+\lambda U\left(x_{n}\right)-g_{n}$ with $g_{n} \in G\left(x_{n}\right), n \geq 1$. Because of hypothesis $\mathrm{H}(f)\left|g_{n}(t, z)\right| \leq \beta_{3}(t, z)+c_{3}\left\|D \tau\left(x_{n}\right)(t, z)\right\|^{p-1}$ a.e. on $T \times Z$, which implies that $\left\{g_{n}(., .)\right\}_{n \geq 1}$ is bounded in $L^{q}(T \times Z)$ and so by passing to a subsequence if necessary, we may assume that $g_{n} \stackrel{w}{\rightarrow} g$ in $L^{q}(T \times Z)$ as $n \rightarrow \infty$. Also from Proposition 3.2 and Krasnoselskii's theorem (see for example Zeidler [16, Proposition 26.7]) we know that $U: L^{p}(T \times Z) \rightarrow L^{q}(T \times Z)$ is continuous. Because $W_{p q}(T)$ embeds compactly in $L^{P}(T \times Z)$, we see that $x_{n} \rightarrow x$ in $L^{P}(T \times Z)$ as $n \rightarrow \infty$ and so $U\left(x_{n}\right) \rightarrow U(x)$ in $L^{q}(T \times Z)$ as $n \rightarrow \infty$. Hence $\left(\left(U\left(x_{n}\right), x_{n}-x\right)\right)=\left(U\left(x_{n}\right), x_{n}-x\right)_{p q} \rightarrow 0$ as $n \rightarrow \infty$. Here by $(., .)_{p q}$ we denote the duality brackets for the pair $\left(L^{p}(T \times Z)\right.$, $\left.L^{q}(T \times Z)\right)$. Also $\left(\left(g_{n}, x_{n}-x\right)\right)=\left(g_{n}, x_{n}-x\right)_{p q} \rightarrow 0$ as $n \rightarrow \infty$. Therefore we deduce that $\lim \left(\left(\hat{A}\left(x_{n}\right), x_{n}-x\right)\right) \leq 0$. But from Proposition 3.6 we know that $\hat{A}($.$) is of type \left(S_{+}\right)$with respect to $D(L)$. Therefore $x_{n} \rightarrow x$ in $L^{P}\left(T, W_{0}^{1, P}(Z)\right)$ as $n \rightarrow \infty$ and so $\tau\left(x_{n}\right) \rightarrow \tau(x)$ in $L^{p}\left(T, W_{0}^{1, p}(Z)\right)$ as $n \rightarrow \infty$. Also by passing to a subsequence if necessary we can say that $D \tau\left(x_{n}\right)(t,.) \rightarrow D \tau(x)(t,$.$) in L^{p}(Z)$ a.e. on $T$ as $n \rightarrow \infty$. Note that for almost all $(t, z) \in T \times Z \quad\left|D \tau\left(x_{n}\right)(t, z)\right| \leq \max \left\{D \varphi(t, z)|, \quad| D \psi(t, z)|, \quad| D x_{n}(t, z) \mid\right\}$, so by the extended dominated convergence theorem we have that $D \tau\left(x_{n}\right) \rightarrow D \tau(x)$ in $L^{p}(T \times Z)$ as 


\section{DIMITRIOS A. KANDILAKIS AND NIKOLAOS S. PAPAGEORGIOU}

$n \rightarrow \infty$. Therefore at least for a subsequence we can say that $\tau\left(x_{n}\right)(t, z) \rightarrow \tau(x)(t, z)$ a.e. on $T \times Z$ and $D \tau\left(x_{n}\right)(t, z) \rightarrow D \tau(x)(t, z)$ a.e. on $T \times Z$ as $n \rightarrow \infty$. So invoking Theorem 3.1 of Papageorgiou [12] we obtain

$$
\begin{aligned}
g(t, z) & \in \operatorname{conv} \varlimsup \\
& \subseteq F(\tau(x)(t, z), D \tau(x)(t, z)) \text { a.e. on } T \times Z .
\end{aligned}
$$

The last inclusion is a consequence of the fact that $\operatorname{Gr} F=\left\{(x, y, v) \in R \times R^{N} \times R\right.$ : $v \in F(x, y)\}$ is closed, which in turn follows from the fact that $(x, y) \rightarrow f(x, y)$ is lower semicontinuous and $(x, y) \rightarrow \bar{f}(x, y)$ is upper semicontinuous (see Chang [3]). Thus $g \in G(x)$ which means that $v \in V(x)$ and so we have proved that $V($.$) is pseudo-$ monotone with respect to $D(L)$.

Claim 2: $G: L^{p}\left(T, W_{0}^{1, p}(Z)\right) \rightarrow 2^{L^{q}(T \times Z)} \backslash\{\emptyset\}$ is bounded (i.e. maps bounded sets to bounded sets).

This is an immediate consequence of the growth condition on $f(x, y)$ (see hypothesis $H(f))$.

Claim 3: $\operatorname{Gr} G=\left\{(x, g) \in L^{p}\left(T, W_{0}^{1, p}(Z)\right) \times L^{q}(T \times Z): g \in G(x)\right\} \quad$ is sequentially closed in $L^{p}\left(T, W_{0}^{1, p}(Z)\right) \times L^{q}(T \times Z)_{w}$.

Let $\left(x_{n}, g_{n}\right) \in G r G$ and assume that $\left(x_{n}, g_{n}\right) \rightarrow(x, g)$ in $L^{p}\left(T, W_{0}^{1, p}(Z)\right) \times L^{q}(T \times Z)_{w}$ as $n \rightarrow \infty$. As in claim 1 at least for a subsequence we can write that $\tau\left(x_{n}\right) \rightarrow \tau(x)$ in $L^{p}(T \times Z)$ and $D \tau\left(x_{n}\right) \rightarrow D \tau(x)$ in $L^{p}\left(T \times Z, R^{N}\right)$ as $n \rightarrow \infty$. Hence as above via Theorem 3.1 of Papageorgiou [12] we obtain that $g(t, z) \in F(\tau(x)(t, z), D \tau(x)(t, z))$ a.e. on $T \times Z$ and so $g \in G(x)$, i.e. $G r G$ is sequentially closed in $L^{p}\left(T, W_{0}^{1, p}(Z)\right) \times L^{q}(T \times Z)_{w}$.

From claim 2 and 3 above, we deduce at once that $V($.$) is bounded and has a$ sequentially closed graph in $L^{p}\left(T, W_{0}^{1, p}(Z)\right) \times L^{q}\left(T, W^{-1, q}(Z)\right)_{W}$.

Claim 4: $\quad V($.$) is coercive$

From hypothesis $\mathrm{H}(\mathrm{a})(\mathrm{iv})$ it follows that

$$
\begin{aligned}
& ((\hat{A}(x), x))=\sum_{k=1}^{N} \int_{0}^{b} \int_{z} a_{k}(t, z, \tau(x), D x) D_{k} x(t, z) d z d t \\
& \quad \geq c_{1} \sum_{k=1}^{N} \int_{0}^{b} \int_{z}\left|D_{k} x(t, z)\right|^{p} d z d t-\left\|\beta_{1}\right\|_{1} \geq \hat{c}_{1}\|x\|_{L \rho(T, X)}^{p}-\left\|\beta_{1}\right\|_{1}
\end{aligned}
$$

for some $\hat{c}_{1}>0$ (recall that $\|D x\|_{p}$ is an equivalent norm on $X=W_{0}^{1, p}(Z)$ ). Also for every $g \in G(x)$

$$
((-g, x)) \geq-\|g\|_{L^{q}(T \times Z)}\|x\|_{L^{p}(T \times Z)} .
$$

Using Young's inequality with $\epsilon>0$ we obtain

$$
\|g\|_{L^{q}(T \times Z)}\|x\|_{L P(T \times Z)} \leq \frac{\epsilon^{q}}{q}\|g\|_{L^{q}(T \times Z)}^{q}+\frac{1}{\epsilon^{p} p}\|x\|_{L P(T \times Z)}^{p}
$$


Moreover from hypothesis $H(f)$ and Minkowski's inequality it follows that

$$
\|g\|_{L q(T \times Z)} \leq\left\|\beta_{3}\right\|_{L q(T \times Z)}+c_{3}\|x\|_{L P(T, X)}^{p-1}
$$

hence,

$$
\|g\|_{L q(T \times Z)}^{q} \leq 2^{q-1}\left\|\beta_{3}\right\|_{L q(T \times Z)}^{q}+2^{q-1} c_{3}\|x\|_{L P(T, X)}^{p} .
$$

Finally from Proposition 3.2 we know that

$$
((U(x), x))=(U(x), x)_{p q} \geq c_{5}\|x\|_{L P(T, X)}^{p}-c_{6}\|x\|_{L P(T \times Z)}^{p-1}
$$

for some $c_{5}, c_{6}>0$. Combining (9) to (13) above we obtain for all $g \in G(x)$

$$
\begin{aligned}
& ((\hat{A}(x)+\lambda U(x)-g, x)) \\
& \quad \geq \hat{c}_{1}\|x\|_{L^{p}(T, X)}^{p}-\left\|\beta_{1}\right\|_{1}-\frac{\epsilon^{q}}{q} 2^{q-1}\left\|\beta_{3}\right\|_{L^{q}(T \times Z)}^{q}-\frac{\epsilon^{q}}{q} 2^{q-1} c_{3}\|x\|_{L^{p}(T, X)}^{p} \\
& -\frac{1}{\epsilon^{p} p}\|x\|_{L^{p}(T \times Z)}^{p}+\lambda c_{5}\|x\|_{L^{p}(T \times Z)}^{p}-\lambda c_{6}\|x\|_{L^{p}(T \times Z)}^{p-1} .
\end{aligned}
$$

First choose $\epsilon>0$ such that $\hat{c}_{1}>\frac{q^{q}}{q} 2^{q-1} c_{3}$. Then having chosen $\epsilon>0$ this way pick $\lambda>0$ large enough so that $\lambda c_{s}>\frac{1}{c^{p} p}$. With these choices of $\lambda, \epsilon$, from (14) we conclude that $V($.$) is coercive.$

Now rewrite problem (8) as the following equivalent abstract operator inclusion

$$
0 \in L(x)+V(x)
$$

By virtue of claims 1 to 4 and Proposition $3.4(L+V)($.$) is surjective which implies that$ problem (15) has a solution $x \in D(L)$. Therefore there exists $x \in W_{p q}(T)$ which solves the auxiliary periodic problem (8).

Claim 5: Every solution $x \in W_{p q}(T)$ of (8) satisfies $\psi(t, z) \leq x(t, z) \leq \varphi(t, z)$ a.e. on $T \times Z$.

Since by hypothesis is $\psi \in \hat{W}_{p q}(T)$ a lower solution of (1) it follows that

$$
\left\{\begin{array}{c}
((\dot{\psi}, w))+((\hat{A}(\psi), w)) \leq((\hat{f}(\psi), w)) \text { for all } w \in W_{p q}(T) \cap L^{p}(T \times Z)_{+} \\
\psi(0, z) \leq \psi(b, z) \text { a.e. on } Z,\left.\psi\right|_{T \times \Gamma} \leq 0
\end{array}\right\}
$$

where $\hat{f}(\psi)(t, z)=\underline{f}(\psi(t, z), D \psi(t, z))$. Also because $x \in W_{p q}(T)$ is a solution of (8)

$$
((\dot{x}, w))+((\hat{A}(x), w))+\lambda((U(x), w))=((g, w))
$$

for some $g \in G(x)$ and all $w \in L^{p}\left(T, W_{0}^{1, p}(Z)\right.$ ). Subtracting (16) from (17) and using 
$w=(\psi-x)^{+}$as a test function we get

$$
\begin{aligned}
& \left(\left(\dot{x}-\dot{\psi},(\psi-x)^{+}\right)\right)+\left(\left(\hat{A}(x)-\hat{A}(\psi),(\psi-x)^{+}\right)\right) \\
& \quad+\lambda\left(\left(U(x),(\psi-x)^{+}\right)\right)=\left(\left(g-\hat{f}(\psi),(\psi-x)^{+}\right)\right) .
\end{aligned}
$$

From the integration by parts formula for functions in $W_{p q}(T)$ (see Zeidler [16, Proposition 23.23]) we obtain

$$
\left(\left(\dot{x}-\dot{\psi},(\psi-x)^{+}\right)\right)=-\frac{1}{2}\left\|(\psi-x)^{+}(b)\right\|_{L^{2}(Z)}^{2}+\frac{1}{2}\left\|(\psi-x)^{+}(0)\right\|_{L^{2}(Z)}^{2} \leq 0 .
$$

Also from hypothesis $\mathrm{H}(\mathrm{a})(\mathrm{iii})$ we see that

$$
\left(\left(\hat{A}(x)-\hat{A}(\psi),(\psi-x)^{+}\right)\right) \leq 0 .
$$

In addition since $g \in G(x)$, we deduce that

$$
\left(\left(g-\underline{\hat{f}}(\psi),(\psi-x)^{+}\right)\right) \geq 0 .
$$

Using (19) to (21) in (18) we obtain

$$
-\int_{0}^{b} \int_{(\psi \geq x)}(\psi-x)^{p-1}(t, z)(\psi-x)(t, z) d z d t \geq 0
$$

hence $\psi(t, z) \leq x(t, z)$ a.e. on $T \times Z$. In a similar way we can show that $x(t, z) \leq \varphi(t, z)$ a.e. on $T \times Z$. So $\tau(x)(t, z)=x(t, z)$ and $u(t, z, x(t, z))=0$. Therefore $x \in W_{p q}(T)$ is a solution of (2).

Now we will show that the set of all such solutions of (2) is compact in $L^{p}\left(T, W_{0}^{1, p}(T)\right)$. To this end let $\left\{x_{n}\right\}_{n \geq 1} \subseteq W_{p q}(T)$ be a sequence of solutions of (2) such that $\psi(t, z) \leq x_{n}(t, z) \leq \varphi(t, z)$ a.e. on $T \times Z$. So $\left\{x_{n}\right\}_{n \geq 1}$ is bounded in $W_{p q}(T)$. Hence we may assume that $x_{n} \stackrel{w}{\rightarrow} x$ in $W_{p q}(T)$ as $n \rightarrow \infty$. Since $W_{p q}(T)$ embeds compactly in $L^{p}(T \times Z)$ and continuously in $C\left(T, L^{2}(Z)\right)$, we see that $x_{n} \rightarrow x$ in $L^{p}(T \times Z)$ as $n \rightarrow \infty$ and $x(0, z)=x(b, z)$ a.e. on $Z$. Also by definition $\dot{x}_{n}(t)+A\left(t, x_{n}(t)\right)=g_{n}(t)$ a.e. on $T$ with $g_{n}(t, z) \in F\left(x_{n}(t, z), D x_{n}(t, z)\right)$. Because of hypothesis $\mathrm{H}(\mathrm{f})$ we may assume that $g_{n} \stackrel{\dddot{a}}{\rightarrow} g$ in $L^{q}(T \times Z)$ as $n \rightarrow \infty$. So $\left(\left(g_{n}, x_{n}-x\right)\right)=\left(g_{n}, x_{n}-x\right)_{p q} \rightarrow 0$ as $n \rightarrow \infty$. Also from the integration by parts formula for functions in $W_{p q}(T)$ (see Zeidler [16, Proposition 23.23]) we know that

$$
\begin{gathered}
\left(\left(\dot{x}_{n}-\dot{x}, x_{n}-x\right)\right)=\frac{1}{2}\left\|x_{n}(b)-x(b)\right\|_{L^{2}(z)}^{2}-\frac{1}{2}\left\|x_{n}(0)-x(0)\right\|_{L^{2}(Z)}^{2}=0, \\
\text { thus }\left(\left(\dot{x}_{n}, x_{n}-x\right)\right)=\left(\left(\dot{x}, x_{n}-x\right)\right) \rightarrow 0 \text { as } n \rightarrow \infty .
\end{gathered}
$$


Therefore we deduce that $\overline{\lim }\left(\left(\hat{A}\left(x_{n}\right), x_{n}-x\right)\right)=0$ and since by Proposition $3.6 \hat{A}$ is of type $\left(S_{+}\right)$with respect to $D(L)$, we infer that $x_{n} \rightarrow x$ in $L^{P}\left(T, W_{0}^{1, p}(T)\right)$ as $n \rightarrow \infty$. Finally as above we can check that $g(t, z) \in F(x(t, z), D x(t, z))$ a.e. on $T \times Z$. Thus in the limit as $n \rightarrow \infty$ we obtain $\dot{x}(t)+A(t, x(t))=g(t)$ a.e. on $T, x(0)=x(b)$ and $g(t, z) \in F(x(t, z), D x(t, z))$ a.e. on $T \times Z$, i.e. $x \in W_{p q}(T)$ is a solution of $(2)$ and so we have proved the compactness in $L^{p}\left(T, W_{0}^{1, P}(T)\right)$ of the solution set in the order interval $[\psi, \varphi]$.

\section{REFERENCES}

1. R. Ash, Real Analysis and Probability (Academic Press, New York, 1972).

2. S. CARL and S. Heikrila, On a parabolic boundary value problem with discontinuous nonlinearity, Nonlinear Anal. 15 (1990), 1091-1095.

3. K.-C. Chang, Variational methods for nondifferentiable functionals and their applications to partial differential equations, J. Math. Anal. Appl. 80 (1981), 102-129.

4. J. Deuel and P. Hess, Nonlinear parabolic boundary value problems with upper and lower solutions, Israel J. Math. 29 (1978), 92-104.

5. J. Diestel and J. UhL, Vector Measures (Math. Surveys, 15, AMS, Providence, Rhode Island, 1977).

6. E. FEIREISL, A note on uniqueness for parabolic problems with discontinuous nonlinearities, Nonlinear Anal. 16 (1991), 1053-1056.

7. E. FeIreisL and J. NoRbURY, Some existence, uniqueness and nonuniqueness theorems for solutions of parabolic equations with discontinuous nonlinearities, Proc. Roy. Soc. Edinburgh 119A (1991), 1-17.

8. V. FILIPpov, Differential equations with discontinuous right hand side, Mat. Sb. 51 (1960), 99-128 (English Trans.: Transl. Amer. Math. Soc. 42 (1964), 199-232).

9. D. Gilbarg and N. Trudinger, Elliptic Partial Differential Equations of Second Order (Springer Verlag, New York, 1977).

10. A. KUFNER and O. J.-S. FučIK, Function Spaces (Noordhoff, Dordrecht, The Netherlands, 1977).

11. J.-L. Lions, Quelques Methodes de Resolution des Problemes aux Limites Non-Linearies (Dunod, Paris, 1969).

12. N. S. Papageorgiou, Convergence theorems for Banach space valued integrable multifunctions, Internat. J. Math. Math. Sci. 10 (1987), 433-442.

13. J. RAUCH, Discontinuous semilinear differential equations and multiple valued maps, Proc. Amer. Math. Soc. 84 (1977), 277-282.

14. C. Stuart, Maximal and minimal solutions of elliptic differential equations with discontinuous nonlinearities, Math. Z. 163 (1978), 239-249.

15. B.-A. Ton, Nonlinear evolution equations in Banach spaces, J. Differential Equations 9 (1971), 608-618. 


\section{DIMITRIOS A. KANDILAKIS AND NIKOLAOS S. PAPAGEORGIOU}

16. E. ZeIDleR, Nonlinear Functional Analysis and its Applications II (Springer Verlag, New York, 1990).

17. V. Zhikov, S. KozLov and O. OLEINIK, G-convergence of parabolic operators, Russian Math. Surveys 36 (1981), 9-60.

Department of Mathematics

UNIVERSITY OF THE AEGEAN

83200 KaRLOVASSI

SAMOS

GREECE
Department of Mathematics National Technical University ZOGRAFOU CAMPUS

15780 ATHENS

GREECE 